\title{
Gastroduodenal Artery Reconstruction as Salvage Procedure for Pancreas Head Ischemia during Transplantation: A Case Report
}

\author{
Raquel Garcia-Roca, Emiliano Astudillo Pombo \\ Department of Surgery, University of Barcelona, Barcelona, Spain \\ Email: raqgar@hotmail.com
}

Received September 14, 2012; revised October 20, 2012; accepted November 7, 2012

\begin{abstract}
Vascularization of the pancreatic allograft depends on the mesenteric and celiac arteries. During procurement inadvertent sectioning of the branches to the head of the pancreas can lead to ischemia and generally there is a need to remove the graft during the same transplant procedure. Knowledge of several unconventional revascularization techniques in the back table and other salvage procedures after reperfusion can be useful in deciding the fate of the graft once ischemia is established immediately after engraftment. We present a case of revascularization of the gastroduodenal artery after reperfusion of the pancreas to improve blood flow to the head of the pancreas that was ischemia after restoring circulation.
\end{abstract}

Keywords: Pancreas Transplantation; Surgical Techniques; Gastroduodenal Reconstruction; Iliac Jump Graft

\section{Introduction}

We present the case of a 48 years old female with Type I Diabetes Mellitus for 35 years with end-organ damage including renal failure, peripheral vascular disease and retinopathy. She underwent preemptive living donor renal transplantation without complications, treated with Tacrolimus, Mycophenolic acid and prednisone $5 \mathrm{mg} / \mathrm{day}$. She presented for pancreas after living-kidney transplanttation (PALK) 6 months from the living donation procedure with a serum creatinine of $0.7 \mathrm{mg} / \mathrm{dl}$.

The donor was a 49 years old female, with a BMI of $21.83 \mathrm{Kg} / \mathrm{m}^{2}$, deceased due to a cerebral bleed, she required 8 minutes of cardiac resuscitation and had short hypotensive periods afterwards; however, she didn't require vasoactive drugs. The donor maintained normal glycemic control without insulin requirements. The cold ischemia time was 510 minutes (8 h, $30 \mathrm{~min})$. The back table reconstruction was following the center's technique, using the distal superior mesenteric artery that had been dissected into the root of the mesentery, rotated around the neck of the pancreas to be anastomosed to the proximal splenic artery stump. The mesentery of the intestine is ligated, leaving approximately $3 \mathrm{~cm}$ from the edge of the uncinate process, in order to safely avoid occluding branches to the pancreas from the superior mesenteric artery.

At the transplant procedure, the graft is implanted into the distal portion of the vena cava and the proximal right common iliac artery. Upon reperfusion, the head of the pancreas and duodenum remained ischemic, despite measures, such increasing the systemic blood pressure and application of warm water. The arterial anastomosis were inspected and thought to be intact, the tail of the pancreas had a bounding pulse with great parenchymal perfusion. The suspicion was that the inferior pancreatoduodenal artery (IPDA) had been ligated during the retrieval procedure and that collateral flow from the dorsal pancreatic and splenic arteries was insufficient to perfuse the head of the pancreas. The gastroduodenal artery (GDA) stump was reconstructed independently, using a donor external iliac jump-graft to the recipient's common iliac artery (Figure 1) above the previous anastomosis. Post-reperfusion of the GDA, the head of the pancreas became pink and the duodenum began contracting. Once adequate vascularization of the graft was confirmed the exocrine secretions were enteric drained at which time the mucosa was visibly well perfused.

Postoperatively, the graft maintained good function, normal glycemic control and serum pancreatic enzymes, with an uneventful clinical course. A Doppler ultrasound with contrast demonstrated perfusion throughout the head of the pancreas and identified both arterial anastomosis. An abdominal CT-scan with contrast demonstrated homogeneous perfusion of the organ and patency of the arterial grafts (Figure 2). The patient was discharged on hospital day 10 and currently has a functioning graft 


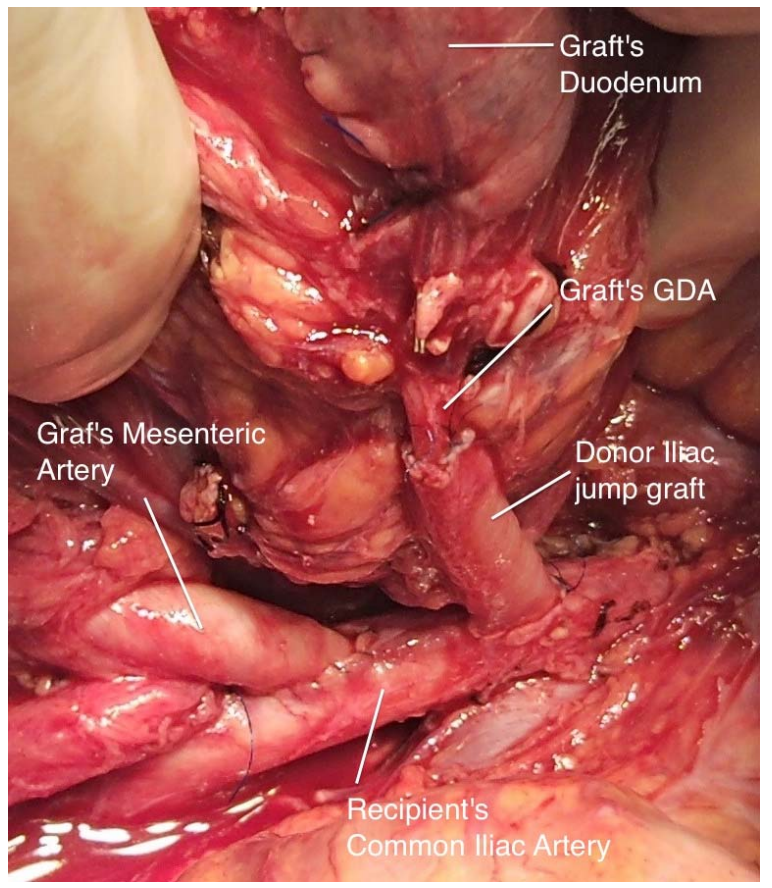

Figure 1. Recipient's common iliac artery anastomosis to the donor jump-graft to the GDA and the pancreas mesenteric artery.

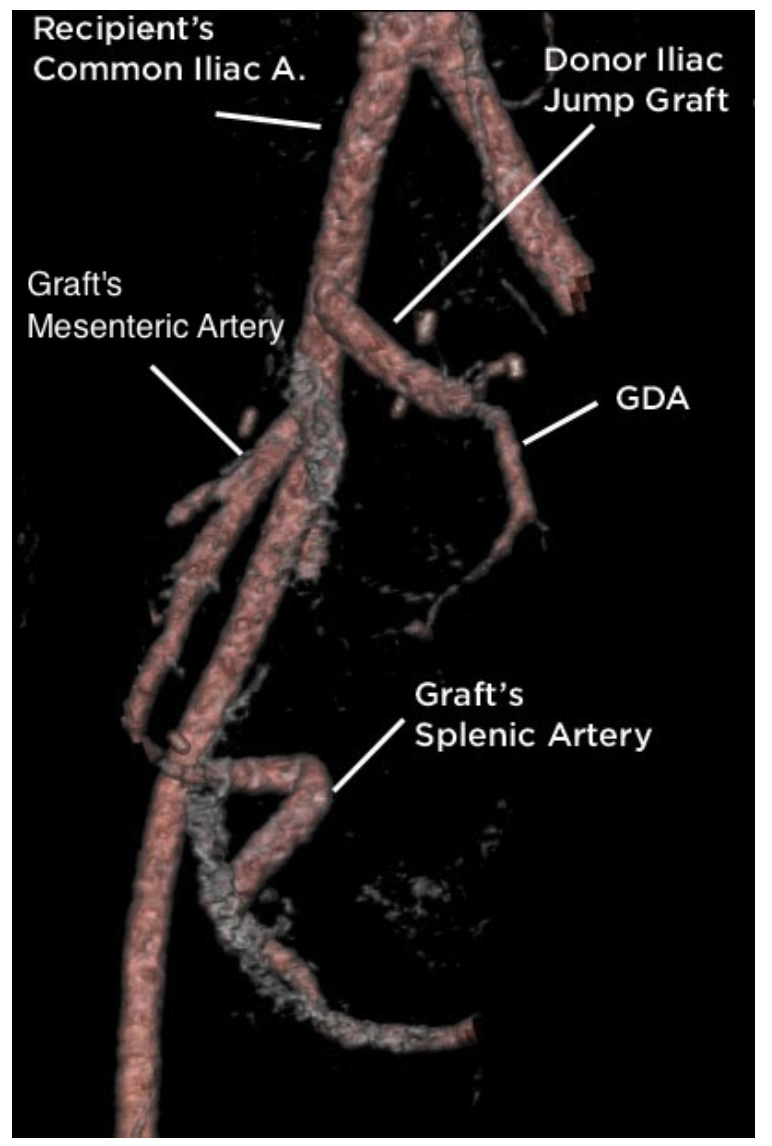

Figure 2. CT angiogram with $3 D$ reconstructions of the arterial supply to the pancreas graft. after 24 months.

\section{Discussion}

The arterial supply to the pancreas is dual, from the celiac trunk and from the superior mesenteric artery [1]. The head of the pancreas irrigation is complex; the GDA out of the celiac trunk splits into the anterior and posterior superior pancreatoduodenal arteries. The first branch from the superior mesenteric artery (SMA) is the IPDA, giving anterior and posterior branches and joining the ones from the GDA, creating the anterior and posterior arcades. The splenic artery and its main branch, the dorsal pancreatic artery, run through the body and tail of the pancreas. Usually collaterals to the head arcades are seen from the dorsal pancreatic artery to the anterior arcade. During a multiorgan retrieval, the celiac trunk is preserved with the liver graft, so the GDA is ligated and the splenic artery is transected at its take off, to be later reconstructed. The IPDA constitutes the main inflow to the pancreatic head and duodenum; it normally takes off within the pancreatic portion of the SMA, in the first 2 $\mathrm{cm}$ of the artery. Sometimes the IPDA might be higher taking off from the first jejunal branch or the mesenteric portion of the SMA; during the retrieval, the intestinal mesentery is ligated and sectioned at the root, a high raising IPDA could be transected inadvertently at the procurement surgery.

Interestingly, collateral flow forms the dorsal pancreatic artery, which might compensate the lack of flow from the GDA, this was seen by the injection of contrast during the back-table preparation of the pancreas graft [2]. Maintenance of good flow to the head of the pancreas is essential since it also perfuses the duodenum; otherwise the intestinal anastomosis might become ischemic and fail, causing peritonitis from enteric leakage. In our case, the head of the pancreas remained ischemic together with the duodenum. Revascularization through the stump of the GDA was performed to improve flow and avoid the consequences of an ischemic anastomosis. Revascularization of the gastroepiploic artery [3] or the gastroduodenal artery [4,5], have been proposed by some groups, to increase blood flow to the head of the pancreas, thus increasing venous return to avoid venous thrombosis. This is done during the bench preparation and considerations are also taken into account during the retrieval process. Using this knowledge, we attempted in situ revascularization of the pancreatic head and duodenum after it became clear that the lack of blood supply to the head would make the transplant unsuccessful.

Other procedures have been used in the event of partial arterial thrombosis, most commonly the splenic artery at the anastomosis, resection of the affected pancreatic parenchyma by distal pancreatectomy can be performed maintaining good graft function. In the case of mesen- 
teric artery thrombosis, head resection can also be performed. The pancreatic duct might be injected with polymers to obliterate the exocrine secretions [6].

Pancreatic head resection was a consideration in this case, but remained a second choice. The experience is that duct injection may cause pancreatitis and pancreatic leak, development of fluid collection and requirement for drainage procedures, all significantly adding morbidity to the procedure [7]. Of course, transplantectomy could have been an option in this case, but less desirable if successful rescue can be achieved.

\section{Conclusion}

The perfusion to the head of the pancreas is mostly dependent on the inferior pancreatoduodenal arcade from the mesenteric artery. This artery can take-off further distal in the mesenteric root than usual and may be ligated during the donor procedure. Some groups, to improve pancreas flow, routinely perform reperfusion of the gastroduodenal artery; however, it can also be used as a rescue technique when the head has poor collateral flow from the splenic territory. This use of the technique after reperfusion has not been previously reported in literature and can be a useful tool to salvage the graft.

\section{REFERENCES}

[1] G. Benoit, H. Bensadoun, M. Moukarzel, C. Gillot and A.
Jardin, "Anatomical Basis of Whole Pancreas Transplantation,” Surgical and Radiologic Anatomy, Vol. 11, No. 3, 1989, pp. 181-185. doi:10.1007/BF02337818

[2] G. Tyden, B. Calissendorff, H. Billing, A. Tibell and R. Linder, "The Vascular Supply in Human Pancreaticoduodenal Grafts: An Angiographic Study,” Transplantation Proceedings, Vol. 22, No. 2, 1990, p. 593.

[3] D. D. Nghiem, "Revascularization of the Gastroepiploic Artery in Pancreas Transplant,” Transplantation International, Vol. 21, No. 8, 2008, pp. 774-777. doi:10.1111/j.1432-2277.2008.00683.x

[4] D. J. Han, "Reconstruction of the Gastroduodenal Artery in Pancreatic Transplantation,” Transplantation Proceedings, Vol. 28, No. 3, 1996, pp. 1727-1728.

[5] N. Thai, A. Khan, K. Tom, A. Basu, R. Shapiro and J. J. Fung, "Revascularization of the Gastroduodenal Artery in a Pancreas Allograft from a Donor with a Replaced Right Hepatic Artery,” Transplantation, Vol. 79, No. 4, 2005, p. 503. doi:10.1097/01.TP.0000145056.82764.53

[6] E. Orsenigo, M. Cristallo, C. Socci, R. Castoldi, P. Fiorina, L. Invernizzi, R. Caldara, A. Secchi and V. Di Carlo, "Successful Surgical Salvage of Pancreas Allograft," Transplantation, Vol. 75, No. 2, 2003, pp. 233-236. doi:10.1097/01.TP.0000041784.27763.A9

[7] T. Konishi, M. Hiraishi, K. Kubota, Y. Bandai, M. Makuuchi and Y. Idezuki, "Segmental Occlusion of the Pancreatic Duct with Prolamine to Prevent Fistula Formation after Distal Pancreatectomy,” Annals of Surgery, Vol. 221, No. 2, 1995, pp. 165-170. doi:10.1097/00000658-199502000-00006 